\title{
Multilinguales
}

\section{L'ingénierie curriculaire en langue française : le modèle de transposition didactique en débat}

Curriculum engineering in French : the model for didactic transposition in debate

\section{Mansour Chemkhi}

\section{OpenEdition}

Journals

Édition électronique

URL : https://journals.openedition.org/multilinguales/5070

DOI : $10.4000 /$ multilinguales. 5070

ISSN : 2335-1853

Éditeur

Université Abderrahmane Mira - Bejaia

Référence électronique

Mansour Chemkhi, «L'ingénierie curriculaire en langue française : le modèle de transposition

didactique en débat », Multilinguales [En ligne], 13 | 2020, mis en ligne le 15 juillet 2020, consulté le 18 mai 2021. URL : http://journals.openedition.org/multilinguales/5070 ; DOI : https://doi.org/10.4000/ multilinguales. 5070

Ce document a été généré automatiquement le 18 mai 2021.

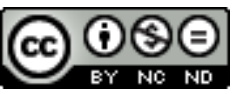

Multilinguales est mise à disposition selon les termes de la Licence Creative Commons Attribution Pas d'Utilisation Commerciale - Pas de Modification 4.0 International 


\title{
L’ingénierie curriculaire en langue française : le modèle de transposition didactique en débat
}

\author{
Curriculum engineering in French : the model for didactic transposition in
} debate

\section{Mansour Chemkhi}

1 La délimitation d'un contenu d'enseignement nécessite l'existence de références extérieures. L'objet à enseigner ne peut fonctionner comme référent à l'égard de luimême. Qu'adviendrait-il de l'école au cas où les pratiques scolaires seraient à ellesmêmes leurs propres références? "Aucun savoir scolaire ne peut être à lui-même sa propre référence. Combien de temps pourrait durer un enseignement de mathématiques qui "créerait » une discipline scolaire non fondée sur des théorèmes reconnus non valides par des mathématiciens " (Joshua: 1998). Pour postuler à une présence significative dans les programmes scolaires, le savoir à enseigner est, donc, tenu d'avoir un référent extérieur. En didactique des langues, les références sont constituées par les descriptions élaborées dans le domaine des sciences du langage ainsi que par les pratiques sociales de la langue, qu'on apprend, dans la communauté linguistique de référence.

Se pose alors le rapport entre l'objet à enseigner et son référent extérieur. Cette relation est, d'évidence, loin de pouvoir être définie en termes d'identité et d'homologie. Le concept de référence traite de la question des écarts toujours présents entre la version scolaire de l'objet à enseigner et sa version de référence. L'objectif de l'école ne peut, aucunement, être celui d'amener les élèves à la maitrise de l'objet à enseigner tel qu'il fonctionne dans la communauté (sociolinguistique ou savante) de référence.

2 La notion de pratiques sociales n'est pas étrangère à la tradition méthodologique en français L2 puisque la méthodologie active par documents authentiques interposés (Puren : 2009) privilégie la référence au texte littéraire comme support principal aux activités d'enseignement. Faire lire des textes issus du domaine de la littérature est, à 
vrai dire, l'activité la plus partagée parmi les méthodes de français notamment en contexte de français langue seconde. Or, on ne peut réduire les pratiques sociales de la langue aux seules pratiques littéraires: les méthodes inspirées du Cadre Européen Commun de Références pour les Langues (désormais CECR ou le Cadre) privilégient de nouvelles pratiques de référence, celles ayant cours dans les circonstances de l'échange ordinaire, voire dans l'espace du discours spontané, au détriment des textes littéraires. Ceci complexifie les tâches d'ingénierie curriculaire relatives tant à l'objectivation de ces nouvelles pratiques de référence qu'à leur didactisation.

Toutes ces questions orienteront le présent article sans en ordonner, pour autant, le plan.

\section{La transposition didactique entre la nécessite d'actualisation des programmes et les approches a tendance applicationniste}

3 La rapidité avec laquelle se renouvelle et évolue la connaissance dans le domaine de la recherche scientifique force l'école à adapter ce qu'elle enseigne à l'évolution des connaissances scientifiques de référence. L'usure du savoir enseigné à l'école diminue la distance entre ce que construisent les chercheurs comme savoirs savants et ce que proposent les enseignants aux élèves comme contenus d'enseignement. Les réformes se produisent sous la pression de l'émergence permanente de nouvelles connaissances dans la cité savante ainsi que sous l'exigence que suscite l'usure du savoir enseigné à l'école. "Avec le temps, le savoir traité par le système d'enseignement vieillit; il apparaît un jour comme vieux par rapport à la société (par rapport au savoir savant et par rapport au savoir banalisé) ». (Chevallard, $1991:$ p. 26)

4 Les curricula de français, pour être adaptés à l'actualité des connaissances qui s'élaborent dans les domaines de la recherche scientifique, sont appelés à s'ouvrir sur l'apport des sciences du langage. Or, la didactique entretient une relation historiquement ambiguë voire problématique avec la linguistique : l'apport que cette discipline fournit à l'enseignement des langues demeure volontairement limité; le souci majeur des méthodologues étant le risque de basculer dans des démarches franchement applicationnistes. Si, par exemple, les enseignants de mathématiques, de physique et de chimie enseignent de la mathématique, de la physique et de la chimie, les enseignants de français, quant à eux, n'enseignent pas de la linguistique. (Galisson, 1989 : 109)

\section{Le modèle de transposition : de la didactique des mathématiques à la didactique des langues}

5 Le modèle de transposition didactique, tel qu'il a été imaginé pour les mathématiques, a eu comme conséquence première celle d'accentuer la subordination des disciplines scolaires aux disciplines académiques de référence. Ce modèle spontanément pensé comme « descendant» (allant des disciplines académiques vers les disciplines scolaires) réduit l'espace de manœuvre de la didactique des disciplines à l'application et limite, par conséquent, sa fonction à la simple transmission du savoir issu des disciplines scientifiques de référence. Fillon fait remarquer «que par tradition la discipline 
académique et la discipline scolaire portent en général le même nom ce qui ne va pas sans engendrer des difficultés ». (Fillon, $2001:$ p. 17). Les contenus à codifier dans les curricula sont évalués par la mesure de la qualité de la transposition : Le savoir, arraché à son contexte originel et déposé dans un autre contexte, a-t-il changé de sens ? La distance séparant le savoir réellement enseigné du savoir savant de départ, conforte-telle, sous l'effet des exigences de simplification, le désagréable sentiment d'une quelconque déformation? Le savoir en question est-il suffisamment actuel ? Etc. (Halté, 1998).

L'une des raisons pour lesquelles les curricula sont réécrits est la nécessité de les adapter à l'évolution de la connaissance scientifique de référence. Nous illustrerons ultérieurement cette idée par des exemples de contenus empruntés à des curricula actuellement en usage en contexte scolaire tunisien.

\section{La transposition didactique : La belle idée d'actualisation des curricula}

6 L'actualité des contenus à sélectionner pour l'enseignement est l'un des critères fondamentaux que les concepteurs des programmes tiennent à prendre en compte lors de la réécriture des syllabus.

«Le développement d'ordre scientifique de la discipline ainsi que celui de ses modèles didactiques correspondants expliquent l'opportunité de réécrire un curriculum en l'actualisant et en l'aménageant en fonction de ces développements qui doivent se traduire selon le processus de la transposition didactique. » (Miled, $2005: 127)$.

7 Le modèle de transposition didactique fournit des démarches transdisciplinaires destinées à modéliser le processus de transformation que subit le savoir en passant de la sphère savante (sphère où s'élabore le savoir académique) à la sphère scolaire (sphère où le savoir est mis en œuvre dans des situations d'apprentissage). La question principale que les didacticiens ont tendance à privilégier, dans la perspective des programmes rédigés en termes de contenus, consiste à s'interroger sur la manière dont un objet scientifique est transformé en objet d'enseignement (décontextualisation, recontextualisation, déformation, simplification). L'on évincera volontairement, de notre réflexion ces aspects inhérents au concept de transposition.

Nous nous pencherons, en revanche, sur deux questions que suscite la référence au modèle de transposition, à savoir l'apport qu'il fournit à l'actualisation des programmes de français ainsi que les limites que l'on formule à son encontre en ce sens qu'il accentue la dépendance de la didactique des langues à la linguistique et qu'il réduit sa mission à la simple transmission des connaissances qui s'élaborent dans le domaine de la recherche scientifique.

8 Par exemple, avec les nouveaux programmes de français en contexte tunisien, une étape a été franchie puisque la grammaire du discours y est reconnue comme objet à enseigner. En effet, les activités grammaticales comportent, outre la référence à la grammaire de la phrase, des références à la grammaire textuelle et à la linguistique du discours: les types d'énonciation, la modalisation, les actes de langage, les types de progression, l'implicite discursif, etc. font l'objet d'un enseignement-apprentissage explicite dans les programmes du secondaire en Tunisie. (Ministère de l'Education: 2005) 
L'énonciation s'apprend-elle, s'enseigne-t-elle? Cette question pourrait paraître saugrenue si l'on se situait du côté de l'enseignement supérieur dans la mesure où, comme le souligne J-M Defays (2001), tout département de langue ou de linguistique qui se respecte compte désormais un cours, voire un programme consacré à l'énonciation, à la pragmatique et/ou à l'analyse du discours. Néanmoins, il ne s'agit pas, dans le cadre de cet article, de discuter de la pertinence de ce genre de cours au sein des formations universitaires, destinées à de futurs spécialistes de la langue (éventuellement de futurs enseignants) mais de leur adéquation aux besoins particuliers d'un public d'élèves en contexte de FL2.

D'ailleurs, l'intérêt de la scolarisation du savoir emprunté à la linguistique du discours fait débat même en didactique du FLM, voire en didactique universitaire. Maingueneau, reconnaissant l'intérêt de la prise en compte de la linguistique du discours comme élément à inscrire dans la formation initiale des enseignants, précise que même au niveau de l'enseignement supérieur, il serait intéressant que l'on mette en œuvre des dispositifs didactiques qui prennent en compte la spécificité de ces contenus. Sa longue expérience de l'enseignement de phénomènes d'énonciation aux étudiants, explique-til, l'a amené à prendre conscience des difficultés que suscite un apprentissage grammatical qui n'est pas centré sur la morphosyntaxe et le lexique. Il s'agit surtout, selon lui, de ne pas sous-estimer la divergence, en termes de ressources mentales, entre les démarches cognitives qu'impliquent respectivement l'étude de la phrase et celle du texte ou du discours. (Maingueneau : 2005)

En d'autres termes, s'il y a bien nécessité d'actualisation des contenus d'enseignement, on peut toutefois se demander si les nouveaux contenus candidats à l'enseignement sont sélectionnés et mis en forme de manière à contribuer au développement des compétences prévues et à servir de ressources réelles susceptibles de favoriser le développement de véritables compétences langagières chez l'apprenant.

\section{Transposition didactique et applicationnisme}

Dans le domaine de l'enseignent des langues, le mouvement descendant caractérisant le rapport de la didactique à la linguistique est fortement critiqué. La linguistique appliquée (terminologie employée initialement pour dénommer ce qu'on allait appeler ultérieurement "didactique des langues») visait essentiellement la scolarisation du savoir élaboré dans le domaine des sciences du langage.

«La question de la transposition didactique est corrélée à celle de ce qu'on a appelé
péjorativement l'applicationnisme : le fait de plaquer directement, sans modalités
de transposition didactique ni a fortiori de questionnement sur les besoins effectifs
d'apprentissage, des savoirs savants dans l'enseignement. Ce phénomène a
notamment été actif en didactique des langues dans les années 1960-70, sous
l'impact d'une linguistique structurale et générative culminante, dont la didactique
des langues et la recherche en didactique étaient positionnées comme de simples
prolongements. Il a été radicalement remis en question dès les années 1980 dans
l'espace francophone.» (Blanchet, $2013: 248$ )

11 Dans la deuxième partie du vingtième siècle, l'objectif de l'enseignement des langues a complètement changé. La langue est envisagée dans sa dimension communicative. Le renouvellement méthodologique qui se met en place alors propose quatre savoir-faire à développer : savoir écouter et savoir parler pour l'oral, savoir lire et savoir écrire pour l'écrit. Les apprenants sont appelés, désormais, à acquérir avant tout des compétences relatives à l'usage de la langue en même temps que des savoirs sur la langue. Il s'agit, en 
fait, d'une véritable remise en question de la place du savoir savant, jugé insuffisant pour garantir une maîtrise pratique de la langue.

Le savoir théorique de type métalinguistique, c'est une banalité de le dire aujourd'hui, ne garantit aucunement l'émergence de véritables capacités communicatives. Le modèle de transposition didactique, du fait qu'il s'applique à des savoirs savants et non aux usages qui sont faits de ces savoirs, devient de plus en plus insuffisant voire inopérant.

"Je suis parfois étonné de voir l'insistance de certains didacticiens du français sur les savoirs, alors qu'ils ont affaire à une pratique qui ne s'appuie pas entièrement et je dirai même pas essentiellement sur des savoirs. » (Perrenoud, 2001:25).

Les nouveaux programmes tendent à poser comme modèle de référence les pratiques sociales du langage, érigées en pratiques de référence.

\section{De la transposition didactique aux pratiques sociales de référence}

13 Considérons, à titre d'exemple, les pratiques linguistiques circulant dans le domaine de la littérature et les usages qui sont faits de la langue dans l'espace médiatique (presse écrite et audiovisuelle). Il s'agit, sans nul doute, de pratiques de référence au sens de Martinand (1986). "A n'en pas douter, elles ont quelque chose à voir avec l'enseignement de français et, puisqu'il est hors de question de les transporter dans l'école, elles doivent certainement être modélisées » (Halté, 1998 : p. 183). Les pratiques langagières de référence pour devenir objet d'enseignement passent par une étape qu'on pourrait appeler de modélisation. Nous désignerons par modélisation l'élaboration de modèles didactiques correspondant aux pratiques langagières sélectionnées pour l'enseignement. On imaginerait mal l'enseignement d'un objet langagier dont on ne sait bien comment il fonctionne dans le milieu social de référence. On imaginerait mal, aussi, la possibilité d'élaboration de modèles didactiques de pratiques langagières sans qu'il y ait référence aux apports de la linguistique pour décrire les produits textuels, produits sémiotiques issus de la communication verbale, avant de pouvoir les modéliser et d'en envisager la codification dans les programmes d'enseignement.

\section{De la modélisation des pratiques sociales de référence en didactique des langues}

14 Le problème de la transposition didactique se complexifie dans le cas de l'enseignement des langues. Les démarches courantes d'ingénierie font usage de la notion de transposition (le cas de la mathématique comme exemple) pour assurer la scolarisation des savoirs savants. On peut recourir aux mêmes démarches dans des disciplines comme la géographie, la biologie, la physique, l'économie et, à la limite, la grammaire (Roulet, 1999). Ces disciplines reposent sur un noyau identifiable de savoirs théoriques à partir duquel il est relativement facile d'élaborer des programmes adaptés à un niveau scolaire et à une filière. Il n'en est pas de même pour l'enseignement des langues: les sciences du langage ne fournissent qu'une partie limitée aux contenus codifiés dans les curricula. La matière verbale spécifiée dans les programmes n'est pas, pour l'essentiel, la transposition de savoirs sur la langue puisque l'objectif n'est pas la 
transmission d'un savoir déclaratif de type métalinguistique mais le développement, chez les élèves, d'un savoir-faire communicatif largement indépendant du savoir savant que les linguistes construisent dans leurs disciplines scientifiques et que la didactique importe dans les classes de langue.

De même qu'un savoir savant ne peut entrer tel quel en classe et que l'objectif de l'enseignement ne peut être la maîtrise de ce savoir tel qu'il fonctionne dans le monde de la recherche, de même que la pratique langagière concernée par l'enseignement/ apprentissage ne pourrait entrer en classe telle quelle et que l'objectif de l'école ne pourrait être celui d'amener les élèves à la maitrise de cette pratique telle qu'elle fonctionne dans la communauté linguistique de référence.

« Pour bien comprendre le rapport entre les objets langagiers travaillés à l'école et ceux qui fonctionnent comme référence, il faut donc, à notre avis, partir du fait que le genre travaillé à l'école est toujours une variante du genre de référence, construite dans une dynamique d'enseignement/apprentissage[...]Il nous semble que la voie empruntée actuellement en didactique pour aborder ce problème peut être décrite par ce que nous proposons d'appeler l'élaboration de modèles didactiques de genres. » (Schneuwly et Dolz, 1997 : 34)

Les pratiques sociales de référence interviennent différemment dans l'espace didactique : elles sont, comme le signale Halté, pourvoyeuses de produits sémiotiques complexes (des textes et non des phrases ou des mots) dont l'exploitation est susceptible d'être envisagée et menée en classe.

« Pour traiter ceux-ci (les produits textuels), dira-t-on, le savoir n'est pas issu nécessairement de la modélisation socio-institutionnelle (bourdieusienne ou autre) mais de savoirs savants d'une autre nature, tirés par exemple de la linguistique textuelle ou de l'histoire littéraire. Les produits textuels alors ne servent que de support à l'enseignement, ils n'en constituent pas la base : l'objet d'enseignement proprement dit n'est pas le produit textuel en lui-même, mais un savoir savant. (....)Cela complique encore la situation, mais le point important n'est pas celui-là : bien qu'il ne faille pas confondre le moins du monde telle modélisation de pratique et tel savoir savant dans la théorie, ils sont cependant solidaires et coactifs dans l'intervention didactique. (Halté, 1998 : 183)

Pour didactiser les textes pris comme modèle de référence, intervient alors la nécessité de les décrire. Le recours à la linguistique du discours devient, dès lors, incontournable. On fait appel au savoir savant issu de la linguistique pour décrire le mode de fonctionnement des produits textuels représentant les pratiques langagières sélectionnés pour l'enseignement, condition nécessaire à leur modélisation didactique. D'ailleurs la légitimité ayant trait à la présence de tels produits langagiers dans les curricula passe par la référence à des savoirs savants ou d'experts (Schneuwly et Dolz, 1997 : p. 34).

En somme, le concept de transposition didactique fournit, certes, un apport limité à une didactique qui vise non seulement la maîtrise du système grammatical mais aussi l'emploi de la langue comme instrument de communication. Il faudrait, néanmoins, admettre que ce modèle ne cesse d'être sollicité dans la conception des programmes étant donné que toute pratique du langage mobilise des savoirs passibles de transposition didactique. 


\section{La modélisation didactique : de l'action sociale à la tâche scolaire}

La langue est un outil qui intervient dans la réalisation de presque toutes les tâches sociales; il n'y a quasiment pas d'activités sociales qui n'aient pas de facettes linguistiques. L'objectif que se donne l'approche actionnelle est de développer chez l'apprenant usager de la langue la capacité de mettre en œuvre ses compétences langagières au service de l'action sociale. Puren (2009) fait la distinction entre l'action sociale (ce que l'on veut que l'apprenant soit capable de faire en langue-culture étrangère en société) et la tâche (ce que l'on fait faire en classe aux apprenants pour qu'ils soient capables de réaliser en société les actions visées). L'action sociale, activité de référence de l'approche actionnelle, présente selon Puren l'objectif de l'enseignement des langues alors que la tâche constitue le moyen pour y parvenir.

Pour préparer l'apprenant à être un acteur social à part entière, on lui fait faire en classe ce que l'on souhaiterait qu'il soit capable de faire plus tard dans la société. A la finalité sociale de l'action, il convient d'opposer la finalité didactique de la tâche. Ce que la notion de tâche permet de transposer est l'activité du sujet, laquelle activité est nécessaire à l'apprentissage. L'activité de l'apprenant engagée dans la réalisation de tâches scolaires imite et reproduit celle de l'acteur de référence engagée dans la réalisation d'actions sociales. Les théories de l'activité dont s'inspire l'approche par les tâches postulent que l'apprentissage est un processus de développement et de construction de connaissances dans lequel l'apprenant est l'acteur central. La notion de tâche permet de transposer en classe ce que les référentiels ne sauront transposer à savoir l'activité du sujet.

La didactique du français L2 a globalement développé deux grandes configurations méthodologiques dont la mise en place s'inspire largement du modèle de pratiques sociales de référence. Selon le type de pratique prise comme référence (pratiques littéraires ou pratique ordinaire de la langue) dans les curricula, les objectifs des formations en langue se distinguent nettement d'une configuration à l'autre.

\section{Le modèle de pratiques sociales de référence : deux exemples d'exploitation en $\mathrm{fl} 2$}

Le concept de pratiques sociales de référence a inspiré les deux plus grandes méthodologies de l'histoire contemporaine de la didactique du français: la méthodologie active par documents authentiques interposés et l'approche actionnelle au sens où ces deux approches sont employées dans Puren (2009). Ces approches, vu les objectifs sous-tendant l'émergence et la constitution de chacune d'elles, sont centrées sur deux types d'usage de la langue. Si l'approche par documents authentiques privilégie la référence aux pratiques linguistiques circulant dans les circonstances de l'échange culturel, l'approche actionnelle repose, quant à elle, sur la référence aux usages linguistiques ayant cours dans la communication courante. Après avoir rappelé les caractéristiques techniques de chacune de ces approches méthodologiques, nous nous attacherons à expliquer les orientations que cultivent l'une et l'autre en termes de références extérieures empiriques. 


\section{Des curricula inspirés de la méthodologie active ordinaire}

21 La méthodologie globaliste ordinaire, ayant selon Beacco (2007) absorbé les principes de l'approche communicative, se distingue par les caractéristiques techniques suivantes :

- Des séquences didactiques longues : les séquences didactiques occupent autour d'une dizaine de pages ;

- Des séquences didactiques à cohésion faible : Ce qui lie les activités les unes aux autres est généralement l'unité thématique ;

- Un nombre d'objectifs assez élevé et de nature différente : on vise à la fois des objectifs relevant des différentes composantes de la compétence communicative (lexique, grammaire, énonciation, textualité, culture, etc.);

- Un support de bonne taille : la séquence didactique a comme seul support principal un texte relativement long, destiné à être exploité dans les différentes activités de classe de langue (compréhension de l'écrit, grammaire, vocabulaire, production de l'écrit);

- Une cohésion faible entre le support et les activités qu'il suscite. Il suffit de la présence d'une seule forme verbale pour justifier une systématisation portant sur tout le paradigme notamment dans le cas des supports authentiques. (Beacco, 2007)

La permanence de l'approche globaliste (confondue avec la version basse de l'approche communicative) est la conséquence $d u$ conservatisme qui est celui de la culture éducative (la résistance au changement) mais surtout la conséquence de la souplesse caractérisant cette approche, laquelle caractéristique la destine à absorber les éléments méthodologiques issus des recherches innovantes en matière de méthodologie d'enseignement sans que ses principes et son organisation interne en soient fondamentalement affectés.

«Elle a pu s'incorporer par exemple, les exercices structuraux, l'approche sémique $\mathrm{du}$ lexique, la méthode verbo-tonale, les activités de créativité, la notion de fonction (acte de langage) et des activités dites interculturelles. L'approche globaliste n'est pas une orthodoxie méthodologique et elle est ouverte à bien des métissages, qui la transforment mais ne la modifient pas. » (Beacco, 2007 : 53).

L'organisation des activités d'enseignement, notamment en contexte tunisien, présente globalement les caractéristiques techniques dont parle Beacco et que nous avons essayé de résumer ci-dessus.

La référence au texte littéraire est liée, en fait, aux objectifs de la méthodologie active par documents authentiques. En effet, cette approche se sert de toute sorte de documents authentiques mais essentiellement du texte littéraire compte tenu de son objectif consistant à former de futurs acteurs sociaux capables de continuer à maintenir un contact à distance avec la langue culture-cible. Il s'agit, précisément, de la méthodologie qui continue à structurer le plus la conception des programmes postprimaires dans les pays du Maghreb dans la mesure où la référence au texte littéraire y reste la référence dominante. Cet objectif de référence, fondé sur le principe de contact à distance avec la langue culture-cible, est en train de perdre de son évidence dans un environnement de plus en plus ouvert à la mobilité internationale. 


\section{Des curricula inspirés du CECR} celle de genre de discours.

«Est définie comme texte toute séquence discursive (orale et/ou écrite) inscrite dans un domaine particulier et donnant lieu, comme objet ou comme visée, comme produit ou comme processus, à activité langagière au cours de la réalisation d'une tâche. » (Conseil de l'Europe, $2001: 15$ ).

Les activités langagières se concrétisent empiriquement à travers des textes. Ceux-ci sont définis comme le produit empirique des pratiques langagières. Dans le Cadre, la notion de texte s'étend aux objets langagiers issus des activités de communication courantes. La communication entre les individus se fait par des textes qui s'organisent en genres discursifs reconnus socialement comme adaptés à une situation communicative donnée (Bronckart, 2008).

L'une des approches les plus circulantes en ce qui concerne l'orientation méthodologique du Cadre est celle qui figure dans Beacco (2007) et Richer (2012). Dans ces ouvrages, les auteurs développent l'idée de l'importance des genres de discours comme catégorie unificatrice des deux composantes centrales de la capacité à communiquer par le langage, à savoir la compétence sociolinguistique et la compétence pragmatique (Beacco, 2007) et comme catégorie susceptible de faciliter la didactisation de la notion floue de tâche (Richer : 2012) .

L'approche préconisée par le CECR privilégie les textes utilisés dans la communication courante au détriment des textes en circulation dans les circonstances de l'échange culturel. Cette approche dite actionnelle se donne comme objectif de préparer l'apprenant à utiliser la langue pour agir dans des situations de communication courantes. On passe de méthodologies centrées sur le recours aux genres littéraires (classés par type de textes : narration, description, dialogue) à des approches centrées sur la référence aux textes à usage courant, classés par genres discursifs.

Pour revenir au concept de pratiques sociales de référence, disons que la notion de genre discursif, sorti par Bakhtine du domaine de la littérature pour s'étendre aux formes de discours en circulation dans la communication ordinaire, permet de rendre plus opérationnel le modèle de pratique sociale. Un genre de discours renvoie à une forme d'énonciation socialement construite présentant des régularités linguistiques et discursives plus ou moins identifiables. Le concept de genre discursif permet de caractériser les pratiques langagières en termes de situations de communication de séquences textuelles, d'actes de langage et de formes linguistiques relativement stables.

L'ouverture aux pratiques courantes des langues, prises en compte par les descripteurs de compétences fournis par le CECR, offre aujourd'hui des pistes didactiques intéressantes. Cette ouverture permettrait de remédier à un problème didactique majeur: celui d'identifier l'objet-langue et de le référer à des pratiques langagières attestées et socialement reconnues. Sahnoun, posant le problème relatif à l'identification de l'objet-langue dans le cas de l'oral, critique les approches didactiques visant cet enseignement. Elles sont, pour le moins, réductrices relativement au poids de cette activité langagière dans la communication courante. De telles approches spécifient, selon lui, des contenus inadaptés, eu égard aux références extérieures qu'il faudrait prendre en compte dans le cadre d'une didactique davantage orientée vers 
l'acquisition de capacités communicatives pratiques que vers la maitrise d'un savoir théorique sur la langue.

« Même, quand on a opté, plus tard, pour l'étiquette: la pratique de l'oral, cet enseignement était resté, lui aussi, inadapté. En effet, ne répondant pas à un contenu défini selon des critères didactiques et scientifiques, et n'étant pas orienté vers une finalité déterminée par une méthode claire, il se réduisait à des exercices ponctuels improvisés, sans efficacité aucune. » (Sahnoun, 2006:48)

Les descripteurs fournis par le CECR inspirent largement les Référentiels pour le Français (Conseil de l'Europe: 2011). Ces documents, destinés à transposer les descripteurs de compétences dans des langues particulières, identifient des pratiques langagières déclinées par activités langagières (réception, production, interaction écrites et orales). Or, l'ouverture aux apports du Cadre et aux documents qui en sont issus devrait éviter d'être inconditionnelle. La modélisation didactique des textes circulant dans la communication courante, textes largement pris en compte par cet instrument de référence, est sujette à des contraintes majeures : peut-on décrire tous les genres discursifs en termes de régularités (linguistiques, textuelles, situationnelles, pragmatiques, etc.) stables ? S'il est possible de décrire et de prendre en compte au sens de l'enseignement des genres tels que la consultation médicale, le fait divers, l'interview, peut-on faire le même travail avec les textes issus des pratiques linguistiques spontanées (conversations personnelles, amicales, familiales - discussions informelles à bâtons rompus, etc.) ? On ne peut introduire dans une classe de langue des produits textuels dont les formes d'organisation échappent à la compétence de description des analystes de discours.

Ce que les didactiques disciplinaires doivent au modèle de transposition didactique est certes un mieux incontestable. Même si certaines disciplines comme les langues s'appuient moins sur des références empruntées au domaine de la recherche scientifique que sur des références issues des pratiques sociales, il n'y a guère de sens à s'opposer à la théorie de transposition dans la mesure où pour amener les élèves à s'approprier des pratiques langagières, on leur fait construire les connaissances nécessaires au développement et à la construction de ces pratiques. Ce que nous avons cherché à mettre en question dans cet article, c'est l'aspect réducteur de certaines approches didactiques: quelques-unes, à tendance applicationniste, sont manifestement enclines à omettre l'idée que des objets langagiers autres que le savoir savant sont enseignables dans des matières comme le français; d'autres, à volonté communicative, réduisent les références en didactique du français aux seules pratiques verbales littéraires comme modèle unique de référence.

\section{BIBLIOGRAPHIE}

Bakhtine, M. (1984), Esthétique de la création verbale. Paris. Gallimard.

Beacco, J-C. (2007), L'approche par compétences dans l'enseignement des langues, Langues et didactique. Paris : Didier. 
Blanchet, Ph. (2013), Guide pour la recherche en didactique des langues et des cultures, Philippe Blanchet \& Patrick Chardenet (coord.). Paris : Editions des archives contemporaines,

Bronckart, J.P. (2008), « Genres de textes, types de discours et “degrés” de langue ». Hommage à François Rastier. Revue Texto. $\mathrm{N}^{\circ} 10-13$.

Chevallard, Y. (1991), « La transposition didactique, du savoir savant au savoir enseigné ». Grenoble : La pensée sauvage.

Conseil de l'Europe. (2001), Cadre européen commun de référence pour les langues : apprendre, enseigner, évaluer, Paris : Didier.

Conseil de l'Europe. (2011). Niveau B1 pour le français, un référentiel. Paris : Didier.

Defays, J-M. (2001), « Didactique de l'énonciation, l'énonciation en didactique », in Texte, revue de critique et de théorie littéraire, Toronto, Canada, n² 27-28, pp. 209-218

Fillon. P. (2001). «Des résultats d'une recherche en didactique à la définition et la mise en situation de contenus de formation ", in Didactique et formation des enseignants . Aster, no 32, p. $15-3$

Galisson, R. (1989). « Problématique de l'autonomie en didactique des langues (contexte français) », in Langue française, $n^{\circ}$ 82. Vers une didactique du français ? pp. 95-115

Halté Jean-François. « L'espace didactique et la transposition », in Pratiques : linguistique, littérature, didactique, $n^{\circ}$ 97-98, 1998. La transposition didactique en français. pp. 171-192

Joshua, S. (1998). « Des « savoirs » et de leur étude : vers un cadre de réflexion pour l'approche didactique ». Année de la Recherche en éducation, p. 79- 97.

Martinand, J-L, (1986). Connaître et transformer la matière, Peter Lang

Maingueneau, D. (2005). «Réflexions sur la « grammaire du discours » au collège », Le français aujourd'hui 2005/1 ( $\left.n^{\circ} 148\right)$, pages $47-54$

Miled, M. (2005). « Un cadre conceptuel pour l'élaboration d'un curriculum selon l'approche par les compétences », La refonte de la pédagogie en Algérie - défis et enjeux d'une société en mutation, Bureau International de l'éducation. Alger : UNESCO-ONPS. Ministère de l'Éducation nationale. Algérie ; pp. 125-136.

Ministère de l'Education. (2005), le français en situation, Manuel scolaire de $2^{\text {ème }}$ année secondaire. Centre National Pédagogique. Tunisie.

Perrenoud, Ph. (2001), « Compétences, langage et communication », in Collès, L., Dufays, J.-L., Fabry, G. et Maeder, C. (dir.) Didactique des langues romanes. Le développement de compétences chez l'apprenant. Bruxelles : De Boeck Duculot

Puren, Ch. (2009), L'approche actionnelle dans l'enseignement des langues. Edition FLE. Maison des langues.

Richer, J-J. (2012), La didactique des langues interrogée par les compétences. Belgique : Éditions E.M.E. et Inter Communications.

Roulet, E. (1999), La description de l'organisation du discours, Paris : Didier

Schneuwly Bernard, Dolz Joaquim, « Les genres scolaires. Des pratiques langagières aux objets d'enseignement. » In : Repères, recherches en didactique du français langue maternelle, $\mathrm{n}^{\circ} 15$, 1997. Pratiques langagières et enseignement du français à l'école. pp. 27-40 ; 
Sahnoun, M. (2006), « Didactique de l'oral : articulation et harmonisation », Le français aujourd'hui, n $154 \mid$ pages 45 à 56

\section{RÉSUMÉS}

Le concept de transposition didactique, lié à la notion de savoir savant, se trouve amené à s'élargir pour répondre aux exigences d'importer à l'école des objets à enseigner qui ne sont pas référés à du savoir académique mais à des pratiques langagières circulant dans l'espace du discours quotidien.

The concept of didactic transposition, linked to the notion of scholarly knowledge, needs to be broadened so as to meet the requirements to import to school things to be taught which are not referred to academic knowledge but to linguistic practices circulating in the space of daily speech.

INDEX

Mots-clés : transposition, savoir, pratiques sociales, tâche, modélisation

Keywords : transposition, knowledge, social practices, task, modeling

\section{AUTEUR}

\section{MANSOUR CHEMKHI}

Université virtuelle de Tunis (ECOTIDI), Tunisie 